\title{
El comercio electrónico como estrategia de negocios para productos de belleza hacia el mercado chileno: Caso de la empresa the well life company
}

\author{
Volumen XIX No (2). Julio-Diciembre 2019. Pág. 67-85
}

ISSN: 0121-1048 IMPRESO ISSN: 2422-3220 EN LÍNEA

\author{
Romero Rubio, Salvador Abraham \\ Universidad Autónoma de Sinaloa \\ Ciudad Universitaria 4, colonia Villa Universidad, \\ Código postal 80010 \\ No. CVU 715360, Salvador.fca@uas.edu.mx \\ Autor corresponsal
}

\author{
Guzmán Lares, Gregorio \\ Universidad Autónoma de Sinaloa \\ Ciudad Universitaria 4, colonia Villa Universidad, \\ Código postal 80010,gregorioguzman@uas.edu.mx
}

\begin{abstract}
Resumen
El comercio electrónico está provocando cambios fundamentales en las empresas y el comercio a nivel internacional, facilitando transacciones y aumentando la interacción entre los consumidores y comerciantes, por lo que las empresas están optando hacia un creciente uso del internet. En esta investigación se analizaron las áreas de oportunidad que generan en el mercado chileno a partir de la elaboración de un plan de negocios de exportación en la comercialización de productos de belleza al mercado chileno, a través de un modelo de negocios electrónico en la empresa The Well Life Company ubicada en la ciudad de Culiacán, Sinaloa, México. El paradigma metodológico abordado en esta investigación fue el cualitativo, descriptivo, como estudio de caso único y se usó la entrevista semiestructurada como herramienta. La empresa debe tomar en cuenta factores como la globalización, índices de uso de internet, normas necesarias y apoyos gubernamentales o privados, así como aprovechar las virtudes del comercio electrónico. Como conclusión, el mercado chileno es técnicamente factible para la exportación de los productos de belleza gracias a su localización, la cultura del uso de los productos de belleza y a las tendencias de compras a tiendas tanto nacionales como internacionales a través del internet.

Palabras clave: comercio internacional, comercio electrónico, plan de negocios, exportación, negocios en Latinoamérica.
\end{abstract}

Códigos JEL: D23, L81, L22, M1, M16

'Citar: Romero Rubio, Salvador Abraham y Guzmán Lares Gregorio (2019). El comercio electrónico como estrategia de negocios para productos de belleza hacia el mercado chileno: Caso de la empresa the well life company. Inquietud Empresarial, 19(2), 67-85. 
El comercio electrónico como estrategia de negocios para productos de belleza hacia el mercado chileno: Caso de la empresa the well life company

\title{
Electronic commerce as a business strategy for beauty products towards the chilean market. Case of the company the well life company
}

\begin{abstract}
Electronic commerce is causing fundamental changes in companies and commerce at an international level, facilitating transactions and increasing interaction between consumers and merchants, which is why companies are opting for an increasing use of the internet. In this research, the areas of opportunity generated in the Chilean market were analyzed from the elaboration of an export business plan in the marketing of beauty products to the Chilean market, through an electronic business model in the company The Well Life Company located in the city of Culiacan, Sinaloa, Mexico. The methodological paradigm addressed in this research was qualitative, descriptive, as a single case study, and the semi-structured interview was used as a tool. The company must take into account factors such as globalization, internet usage rates, necessary standards and government or private support, as well as take advantage of the virtues of electronic commerce. In conclusion, the Chilean market is technically feasible for the export of beauty products thanks to its location, the culture of the use of beauty products and the trends in purchases from both national and international stores through the internet.
\end{abstract}

Keywords: international trade, e-commerce, business plan, exportation, business in Latin America.

JEL Codes: D23, L81, L22, M1, M16

\section{INTRODUCCIÓN}

Las tecnologías del comercio electrónico están provocando cambios elementales sin antecedentes en el mercado dado que a diferencia de las tecnologías comerciales del siglo XX, el comercio electrónico permite la interactividad, lo que significa que se facilita la comunicación entre los comerciantes y consumidores, mientras que anteriormente otras tecnologías transformaron la vida económica durante ese siglo; ahora la evolución del internet y otros avances tecnológicos están moldeando la del siglo XXI, por lo cual la razón del interés específico en el comercio electrónico es que esta técnica es diferente y más poderosa que las vistas en el siglo anterior (Laudon \& Traver, 2014).
A pesar de los tropiezos en los inicios del comercio electrónico, según Laudon \& Traver, (2014) con la información que obtuvieron de eMarketer Inc en el 2012 y de Internet Retailers, en ese mismo año se esperaba que las ventas en línea al menudeo en Estados Unidos crecieran más de 15\%, en comparación con el $3.4 \%$ tradicional. Se esperaba que alrededor de 150 millones de usuarios de internet hicieran por lo menos una compra durante el año, y 35 millones más usaran la web para recopilar información sobre compras potenciales de productos, mientras que en Europa el comercio electrónico de negocio a consumidor (B2C) crecía más del 18\%, equivalente a $\$ 260$ mil millones, en comparación con las ventas tradicionales, en Brasil, por ejemplo, aumentaron un $20 \%$, en India un $40 \%$ y en China un $140 \%$. 
Como complemento, de acuerdo con Sanou (2015, p. 3), director de la Unión Internacional de Telecomunicaciones (UIT), menciona que "La Agenda Conectar 2020 pretende lograr que al menos el 55\% de los hogares de todo el mundo tengan acceso a Internet en 2020, en relación con el $46.4 \%$ estimado en 2015". También que al menos el $60 \%$ de las personas físicas en todo el mundo utilicen Internet en el año 2020 y serán necesarias iniciativas políticas adicionales para promover una mayor penetración de esa tecnología, a fin de alcanzar el objetivo.

En México la población aproximada en el 2015 era de 121,736,809 personas, de las cuales 60,000,000 tenían acceso a internet, lo que representaba el $49.3 \%$ de la población (Internet World Stats, 2015). El gasto trimestral promedio en todos los dispositivos y categorías, que no se relacionan a viajes, fue de MXN \$5,575.00, alrededor de MXN $\$ 1,860.00$ gastados online por mes (AMIPCI,2015).

Sobre el asunto referente al otro país de interés de esta investigación, que es el país chileno, se agrega que de acuerdo al más reciente estudio sobre Comercio Global Conectado, realizado por The Nielsen Company (2016), en Chile, 8 de cada 10 personas encuestadas en línea (82\%) han comprado alguna vez por internet y dice que "Los brasileños son más propensos a comprar en línea y en segundo lugar se encuentran los chilenos quienes compran más entradas para eventos (49\%), videojuegos (33\%), muebles/decoración (29\%) y domicilios en restaurantes (28\%)".

Para seguir con una estrategia de comercio electrónico la empresa debe entender que el internet es una herramienta y que antes de la adhesión a una solución tecnológica, una evaluación de productos, clientes, situación competitiva, recursos y operaciones de la empresa son esenciales para entender mejor cómo todos esos elementos se integran a una estrategia de comercio electrónico. (Hutt y Speh, 2010, citado en do Amaral, de Camargo, Pizzinatto, \& de Toledo, 2015, p. 228)

Vivimos y trabajamos en una era digital en la que el poder recae en el consumidor lo que significa que ahora los compradores identifican lo que valoran y a quién quieren comprarle, por ello si se les escucha con cuidado se pueden convertir de compradores únicos a leales a largo plazo (Bergiel, Bergiel, \& Bergiel, 2014, p. 31).

Los analistas estiman que para el año 2016, las empresas gastarían unos $\$ 5700$ billones y los consumidores cerca de $\$ 542$ mil millones en tracciones en línea. También estiman que el comercio electrónico para el 2020 representará alrededor de $17 \%$ de todas las ventas al menudeo (Deatsch, 2010, citado en Laudon \& Traver, 2014, p. 14)

Es importante subrayar que el Internet ha generado ventajas en la reducción de costos de transacción para el manejo y aprovechamiento de la información, así como el volumen de información ha crecido exponencialmente, por estas razones el internet como herramienta tiene gran potencial en el desarrollo de distintas actividades económicas y sociales, siendo las relacionadas con el comercio y los negocios una de las más importantes porque ofrecen a la empresa ventajas competitividad en el mercado (Zárate, 2015, p. 38).

Entre los beneficios percibidos son "Aumentar la satisfacción de los consumidores, entender las preferencias del cliente, reducir inventario, rotación de inventario, disminuir las situaciones de falta de existencias y mejorar el tiempo de respuesta y el tiempo de salida al mercado" (Barua, Konana, Whinston, \& Yin, 2004, p. 
El comercio electrónico como estrategia de negocios para productos de belleza hacia el mercado chileno: Caso de la empresa the well life company

586) además que posibilita el cambio de información entre clientes y proveedores, permitiendo a las empresas aumentar la precisión del procesamiento de sus transacciones, más que simplemente el hecho de intercambiar productos o servicios por dinero, beneficiando a todos los envueltos en la cadena (Trepper, 2000, citado en do Amaral et al., 2015, p. 227).

\subsection{Problema de investigación}

El presente proyecto se delimita exclusivamente a la instrumentación de un plan de negocios de exportación para la empresa The Well Life Company S. de R. L. de C. V., productora de productos de belleza, ubicada en la ciudad de Culiacán, Sinaloa, que está categorizada dentro de las microempresas según Nafin (2008) y a la implementación de estrategias para la comercialización de productos de belleza en el mercado chileno, que a su vez puede ser de ayuda para las empresas que presenten características similares tanto a nivel nacional como internacional en tiempo venidero.

Por lo tanto, objetivo de este trabajo consiste en elaborar un plan de negocios de exportación en la comercialización de productos de belleza al mercado chileno, a través de un modelo de negocios electrónico en la empresa The Well Life Company.

Atendiendo esta necesidad, se considera de sumo valor el estudiar y presentar una opción para alcanzar un mercado de exportación y, con ello, establecerse competitivamente en este mercado como una de las principales empresas electrónicas comercializadoras de productos de belleza consolidando una empresa virtual por la cual comenzar su actividad comercial y económica en el mercado chileno para cubrir la necesidad de los consumidores a los cuales va dirigido.

Consecuentemente al conocer las tendencias de salud, belleza y las de los mercados digitales, así como de los rápidos cambios en la era de la información, un efectivo plan de negocios podría ser el factor determinante del éxito de esta empresa.

El comercio electrónico no es una solución para todas las organizaciones dado que un proceso mal conducido puede llevar a un desastre financiero y estratégico creando más problemas que beneficios, no siendo una opción que se pueda seguir a la ligera (Varajão, 2002, citado en Gonçalves et al., 2008).

El presente estudio, en primer lugar, se presenta el contexto tanto internacional como nacional en relación con el comercio electrónico y la exportación de productos de belleza. En segundo lugar, se presentan las teorías de administración, comercio internacional y comercio electrónico que se utilizaron como base para esta investigación.

En tercer lugar, se presenta la metodología utilizada, la cual al ser un estudio de caso único se considera dentro del método cualitativo y se hace uso de una entrevista semiestructurada al propietario de la empresa, además de revisión de documentos y materiales otorgados por la empresa y la revisión bibliográfica de diversas fuentes bibliográficas como la de Conricyt, Google Scholar y libros relacionados con el tema.

La empresa debe tomar en cuenta factores como, el poder adquisitivo, además de documentación necesaria para prevalecer y ser competitiva, a su vez, certificaciones y las tendencias del mercado, así como aprovechar el comercio electrónico porque gracias a éste se evitan costos y no hay 
barreras geográficas. Como conclusión, el mercado chileno es técnicamente factible para la exportación de los productos de belleza gracias a que su localización geográfica, cultura y nicho de mercado.

\section{MARCO TEÓRICO}

Previo a adentrarse en el marco teórico del estudio de la comercialización de productos de belleza al mercado internacional por medio del comercio electrónico, se mencionaron algunos estudios recientes relacionados al tema.

En primer lugar, Romero (2015) en su obra titulada Cambio organizacional para el desarrollo exportador en la mediana empresa hortícola del estado de Sinaloa, en la que se utilizó el enfoque cualitativo con una estrategia de estudio de caso, la muestra se seleccionó de acuerdo a características específicas como que fueran empresas sinaloenses, exportadoras, con más de 10 años en el mercado, entre otras, utilizando entrevistas semiestructuradas, análisis de documentos y observación no participante como instrumentos de recopilación de datos.

En su investigación se enunció que la ausencia de capacitación de los empleados y falta de visión de los dueños de las PyMES hortícolas sinaloenses que efectúan actividades de exportación, ocasiona una falta de competitividad en este sector a nivel nacional y en la comercialización al mercado internacional, al mismo tiempo la ausencia de canales de comercialización, barreras no arancelarias y un sector desarticulado inhiben la libre competencia entre sus competidores norteamericanos, por lo que afirma que para penetrar los mercados internacionales se necesita planeación en cuanto a la logística, negociación, conocimiento de regulación aplicable, entre otros factores como obtención de créditos y certificados de calidad.

Se observó que en la organización tomada como base para el estudio de esta autora, se aplicaron diversos cambios estructurales, nuevas formas organizacionales, un organigrama funcional para mejor comunicación entre las áreas de la empresa, cambios culturales, así como también se observaron mejoras en cuanto a la tecnología mejorando los procesos administrativos de las diferentes áreas de la organización y los productivos, entre otras prácticas que le han permitido a esta empresa incursionar en mercados de exportación y competir en Florida principalmente.

Por su parte Contreras (2015) en su obra titulada La importancia de un plan de negocios de exportación de una empresa de sistemas de riego para el mercado de Costa Rica, Centroamérica. Caso de la empresa Netafim México S.A. de C.V., en la cual se utilizó una metodología predominante cualitativa- descriptiva utilizando algunos datos cuantitativos, determinando como muestra a diferentes gerentes de las diversas áreas de la empresa, que a su vez en el trabajo de campo se realizaron entrevistas no estructuradas e informales, análisis bibliográfico de la empresa, observación de las instalaciones, estructura organizacional, procesos, entre otros.

Las conclusiones a las que se llegaron con este trabajo investigación para la exportación de mangueras originarias de Reynosa, Tamaulipas hacia San José, Costa Rica mediante los trámites y requerimientos en el contexto del TLC entre México y ese país, es que el contexto internacional exige a las empresas mayor preparación de los procesos de internacionalización y que este plan de negocios se puede utilizar como herramienta para enfrentar esos escenarios, dado que 
El comercio electrónico como estrategia de negocios para productos de belleza hacia el mercado chileno: Caso de la empresa the well life company

existen muchas empresas con potencial para exportar porque ya tienen un producto y análisis del mercado pero no se encuentran preparadas para incursionar en el mercado internacional.

Asimismo, menciona que la planeación de la empresa permitió crear estrategias, pensar en oportunidades, tomar decisiones respecto a información financiera, elaborar procesos de exportación, así como un buen control y desarrollo de la empresa y que los elementos para tomar en cuenta para la exportación son la distribución, barreras no arancelarias cualitativas, documentación para exportar, clasificación arancelaria, normas y controles de calidad.

Luego, se analizó el estudio desarrollado por Bernal (2015) en su obra Plan de negocios como factor determinante de crecimiento para una comercializadora de productos deportivos, caso de la empresa Feler Euro-Asian, que utilizó una metodología predominantemente cualitativa-descriptiva con datos cuantitativos por presentar un panorama actual llevando a cabo el proceso de exportación de productos deportivos al mercado de Los Ángeles, California, tomando en cuenta el tamaño del sujeto de estudio al gerente de la empresa quien estaría interactuando y aplicando los diferentes instrumentos de recolección de información, siendo estos entrevistas no estructuradas y encuestas a las diferentes áreas de la empresa, análisis bibliográfico de la empresa, observación de las instalaciones, estructura organizacional, procesos, entre otros.

Este autor menciona que las empresas dedicadas al comercio de ropa y productos deportivos representan un rol importante para la economía mexicana, por ello se considera conveniente la creación de un plan de negocios de exportación hacia Estados
Unidos para las empresas de este giro, dado que aparte de ser de beneficio para la economía de la empresa y del país, es beneficioso para los ciudadanos de ambos países porque fomenta combatir el problema social representativo que es la obesidad.

Concluyó que debido a la creciente competencia en los mercados internacionales, las empresas han estado adoptando nuevas tecnologías para el intercambio de bienes, nuevas maneras de comercialización y nuevos países a los cuales pueden incursionar, por lo cual el plan de negocios es una herramienta indispensable para cumplir estos objetivos dado que ofrece un análisis de mercado y financiero, estrategias de comercialización del producto, oportunidades de negocios potenciales en mercados externos así como evitar riesgos y con todo esto la creación de ventajas competitivas.

Finalmente, se encuentra la investigación denominada: Mercadotecnia digital: estrategia de competitividad en el comercio electrónico de la empresa multinacional. Caso Coppel S.A. de C.V. elaborada por Valdez (2015) en la que se determinó llevar a cabo una investigación documental y de campo de carácter cualitativa, utilizando la entrevista en profundidad semiestructurada para la recolección de datos y de observación no participativa al principio de la investigación que más adelante se le presentaron oportunidades pudiendo así realizar la observación de forma participante.

El mismo autor menciona que la mercadotecnia es un motor para la creación de la demanda de un producto para generar ventas e ingresos, la cual es utilizada por las empresas sinaloenses para la búsqueda de estrategias de competitividad y al utilizarla digitalmente favorece al desarrollo del 
comercio electrónico para alcanzar los objetivos de la empresa multinacional, lo que favorece la comunicación con los clientes así creando fidelidad con la marca de la empresa y con ello establecer relaciones más sólidas con ésta generando así el crecimiento del ecommerce.

A manera de conclusión, señaló que la mercadotecnia digital está siendo utilizada como estrategia de competitividad para establecer una posición rentable y sustentable en el departamento de ecommerce, el cual afirma la mercadotecnia digital apoya a promover el comercio electrónico en el sitio web de la empresa generando ventas, enlazar al cliente con la marca posicionándola, lo cual impacta favorablemente en el desempeño del comercio electrónico; usar las redes sociales como foro para servicio al cliente, así como los motores de búsqueda como parámetro de prestigio y confianza de la marca, y finalmente que la empresa estudiada le está apostando a la tecnología con el afán de que en el futuro ésta logre generar utilidades.

Sin embargo, para lograr esto se necesita la capacitación del personal para que esté actualizado en el mundo digital con la finalidad de encontrar las necesidades cambiantes y tendencias en el mercado.

\subsection{Teoría de la administración}

Para comenzar a presentar la teoría de la administración, se considera importante empezar retomando sus antecedentes, los cuales de acuerdo con (Chiavenato, 2014, p. 37):

" $A l$ despuntar el siglo $X X$, dos ingenieros desarrollaron los primeros trabajos sobre administración. Uno de ellos fue el estadounidense Frederick Winslow Taylor, creador de la llamada Escuela de la Administración Científica, que buscaba aumentar la eficiencia de la industria por medio de la racionalización del trabajo del obrero, El otro, el francés Henri Fayol, desarrolló la llamada teoría clásica, la cual pretendía aumentar la eficiencia de la empresa debido a su forma de organización y la aplicación de los principios generales de la administración con bases científicas."

Mientras que Chiavenato (2014) señala que "La administración es fundamental para conducir la sociedad moderna; no es un fin en sí misma, sino un medio para conseguir que las cosas se hagan de la mejor manera, al menor costo y con mayor eficiencia y eficacia” (p. 13).

Da Silva (2004) menciona a diferentes autores importantes que han sentado las bases de la administración como se conoce hoy en día, tales como a: Adam Smith (17231790) quien "Otorgó gran importancia a la división del trabajo y a sus beneficios y, en su siglo, se anticipó al enfoque de la descomposición de un servicio o una tarea"; Robert Owen (1771-1858) quien "Desarrolló un mecanismo especialmente singular, el "monitor silencioso", para obtener mayores disciplina y motivación. También menciona que Charles Babbage (1972- 1871) describió con detalle las herramientas y las máquinas, expuso los "Principios económicos de la fabricación” y, analizó las operaciones, los tipos de habilidades involucrados y el costo de cada proceso, además de sugerir instrucciones para mejorar las prácticas.

\subsection{Teoría de la administración estratégica}

Ligada a la teoría de la administración, se encuentra la teoría de la administración estratégica, la cual David (2013, p. 5) argumenta que:

"La administración estratégica se define como el arte y la ciencia de formular, implementar y evaluar decisiones multidisciplinarias que permiten que una empresa alcance sus objetivos. 
El comercio electrónico como estrategia de negocios para productos de belleza hacia el mercado chileno: Caso de la empresa the well life company

Como lo sugiere esta definición, la administración estratégica se enfoca en integrar la administración, el marketing, las finanzas y la contabilidad, la producción y las operaciones, la investigación y el desarrollo, y los sistemas de información, para lograr el éxito de una organización."

De acuerdo con Dess, Lumpkin, y Eisner (2011, p. 9):

"La administración estratégica consiste en los análisis, las decisiones y las acciones que emprende una organización para crear y sostener sus ventajas competitivas. Esta definición capta dos elementos principales que llegan al centro de la administración estratégica."

El primero es que la administración estratégica de una organización supone tres procesos permanentes: el análisis que incluyen la misión, visión y objetivos estratégicos, así como los entornos internos y externos; las decisiones estratégicas tomadas por los líderes y acciones que deben emprenderse y para ello se asignan los recursos necesarios y la planeación que permitirá realizar las estrategias. El segundo elemento es que la administración estratégica se enfoca en estudiar por qué algunas empresas tienen mejor desempeño que otras.

Hitt et al. (2015), indica que el entorno de la industria son los factores que influyen en la compañía, sus acciones y respuestas competitivas, que complementado con lo que menciona Porter (2008) este entorno está compuesto por diversos factores como los competidores, clientes y proveedores, conocidos como las cinco fuerzas de Porter, los cuales tienen suma importancia para la estrategia de una empresa.

\subsection{Teoría del comercio internacional}

Torres (2005) menciona que la vida económica capitalista está constituida por personas que producen mercancías con fines lucrativos, mediante los cuales los consumidores satisfacen sus necesidades. El comercio se halla presente en todos los aspectos de la vida como en el trabajo, el comercio de mercancías o divisas, así como en el dinero, que para poder efectuarlas implica tener una oferta y una demanda de esos bienes.

Continuando con el mismo autor, él indica que: "el comercio es la rama de la actividad económica que se refiere al intercambio de mercancías y de servicios, mediante trueque o dinero, con fines de reventa o para satisfacer necesidades". Quién también sostiene que el comercio internacional es un caso especial el comercio general que a pesar de que sufre ciertas modificaciones y complicaciones por causa del tipo de cambio, aranceles y mayores costos de transporte, no cambia su naturaleza de fines lucrativos y de abastecer los que carece otro país.

Los párrafos anteriores sirven como base para comenzar a comprender la evolución del comercio con el paso del tiempo (ver figura 1), que referente a esta investigación es de suma importancia para conocer cómo ha influido hasta llegar a los métodos más actuales de comercio internacional por medio del comercio electrónico, como se continúa explicando más adelante.

\subsection{Teorías tradicionales del comercio internacional}

Explica Krugman et al., (2012), que David Ricardo introdujo la teoría de la ventaja comparativa, la cual ocurre cuando el comercio internacional se basa sólo en las diferencias de productividad del trabajo, conocida como el modelo ricardiado, 


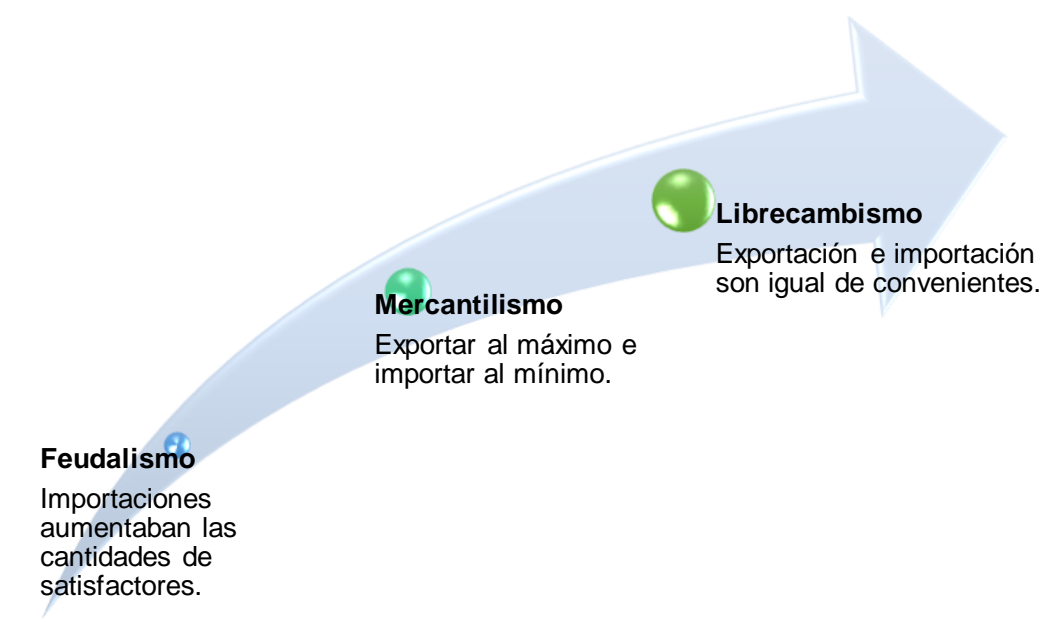

Fuente: Elaboración propia con datos de Torres (2005)

mientras que la teoría de Adam Smith implementa la ventaja absoluta, la cual ocurre cuando se produce un bien con menos trabajo que otro país.

Sin embargo, la ley de la ventaja comparativa puede ser explicada en términos de la teoría del coste de oportunidad introducida por G. Haberler (1936, citado en González (2011, p. 106), la cual establece que el coste de oportunidad es todo lo que se sacrifica de un segundo bien para poder obtener otro bien en su lugar, no solo tomando en cuenta el factor trabajo sino varios factores, concluyendo que el país con coste de oportunidad más alto tiene desventaja comparativa de ese bien mientras que el coste más bajo tiene la ventaja comparativa de ese bien.

Por otra parte Cardozo, Chavarro y Ramírez (2007, p. 5) mencionan que John Stuart Mill observó que las exportaciones varían con los términos de intercambio y la disposición de cada país para exportar dependía de la cantidad de importaciones que obtendría de regreso.

\subsection{Modelo de Heckscher-Ohlin (H-O)}

A pesar de lo expuesto anteriormente González (2011) estipula que: "La teoría de la ventaja comparativa no explica por qué los costes relativos difieren entre los países" (p. 108). Por ello menciona que la aportación de los economistas suecos Eli Heckscher, en 1919, y Bertil Ohlin, en 1933 influye decisivamente para determinar la causa del comercio exterior, dado que el teorema $\mathrm{H}-\mathrm{O}$ postula que una nación exportará el bien que utiliza intensivamente su factor relativamente abundante e importará el escaso.

González (2011) cita que por una parte Heckscher afirmaba que como requisito para que se efectúe el comercio internacional, los países tienen distintos precios relativos de los factores de producción y diferentes proporciones de factores productivos para diferentes bienes, mientras que B. Ohlin insistía que la distinta dotación factorial es la causa fundamental de las diferencias de los costes comparativos.

Con la teoría $\mathrm{H}-\mathrm{O}$ se agrega un ingrediente más a las causas del intercambio de bienes y servicios entre las naciones que 
El comercio electrónico como estrategia de negocios para productos de belleza hacia el mercado chileno: Caso de la empresa the well life company

toma en cuenta los factores relativos de producción de los bienes entre los países, avanzando así en la historia del comercio internacional para llegar a la nueva teoría moderna que se aborda a continuación.

\subsection{La nueva teoría sobre el comercio internacional}

Para González (2011), la teoría clásica del comercio internacional no alcanza a explicar fenómenos actuales porque anteriormente primero se creaban las teorías que seguidamente eran contrastadas empíricamente mientras que en la nueva teoría sucede lo contrario, por lo que se desprenden teorías alternativas.

El mismo autor menciona que por medio de la ventaja comparativa el comercio internacional toma un papel del tipo interindustrial, esto es, los países importan y exportan los productos de diferentes industrias $y$ se incrementará cuantos mayores sean las diferencias, que por contraparte el comercio intraindustrial se realiza entre países similares.

Otro rasgo destacado del comercio internacional destacado por el autor antes mencionado es el del peso que tienen las multinacionales, dado que productos de empresas que tienen grandes cuotas de mercado, como la tecnología, farmacéuticos y automóviles, implican un contexto de competencia imperfecta.

Entendiendo con todo lo anterior que los países toman en cuenta diferentes factores como sus similitudes, distancias y la influencia de las multinacionales, así como los tipos de productos, además de las diferencias entre las naciones, lo cual conlleva a un panorama más claro de cómo el comercio internacional ha ido evolucionando a través del tiempo para con ello comprender las razones por las cuales los países intercambian productos y servicios entre ellos y prepararse a los cambios que ocurren recurrentemente.

\subsection{Enfoque Born Globals}

Diversos autores como Oviatt y McDougall (1994, citado en Madsen \& Servais, 1997), han definido a las Born Globals como: “Una organización de negocios que, desde su inserción busca obtener una significativa ventaja competitiva en el uso de sus recursos y la venta de sus productos en múltiples países" (p. 563), también conocidas como International New Ventures (INVs).

Baier-Fuentes et al., (2019) mencionan que Rennie (1993) llama a este tipo de empresas International New Ventures (INVs), mientras que Oviatt y McDougall (1995) las conocen como Global Start-ups y que Fillis (2001) las nombra como Instant Internationals.

Según Baier-Fuentes et al. (2019) algunas de las dimensiones que se usan comúnmente para esta área incluyen el porcentaje de ventas al exterior, el tiempo, el alcance geográfico y el tipo de industria a la que pertenecen. Recientemente, se aclararon con base en la literatura las posibles diferencias entre BG e INV, señalando que el modo de entrada en los mercados extranjeros es una de las principales diferencias (Baier-Fuentes et al. 2019). Aun así, se analiza la confusión terminológica a través de cuatro criterios: el porcentaje de ventas al exterior del $25 \%$, la velocidad de internacionalización midiendo el tiempo entre la fundación de la empresa y el primer año de la internacionalización, el alcance con respecto a los mercados penetrados y el cuarto criterio q se utiliza para clasificar rápidamente a las empresas que se internacionalizan es el sector industrial en el que operan. 
Se puede constatar que el aumento de Born Globals puede atribuirse a por lo menos tres factores importantes según Madsen y Servais (1997, p. 565) con base en la literatura, los cuales están interrelacionados y son: las condiciones de nuevo mercado, las capacidades más elaboradas de personas, entre ellas el fundador o empresario $\mathrm{y}$ finalmente, los avances tecnológicos en las áreas de producción, transporte $\mathrm{y}$ comunicación.

\subsection{Modelo e-Commerce}

Para alcanzar una mejor comprensión del modelo de e-commerce, primero se define lo que es un negocio electrónico tomando lo mencionado por los autores Sawhney y Zabin (2001) quienes indican que es: "El uso de las redes electrónicas y las tecnologías asociadas para habilitar, mejorar, optimizar, transformar o inventar un proceso de negocio o sistema de negocio para crear un valor superior para los clientes actuales o potenciales" (p. 15, citado en Palacios et al., 2014, p. 227).

En otras palabras, un negocio electrónico, es una empresa que utiliza la tecnología para llevar a cabo los procesos de las distintas áreas funcionales colaborando entre ellas de una manera más eficiente, de este modo mejorando las áreas, creando así una ventaja para el negocio. El área de e-commerce se encuentra dentro del negocio electrónico o bien, de un negocio tradicional, por ello se considera importante abordar a continuación el modelo del comercio electrónico.

Laudon y Traver (2014) definen al comercio electrónico como el "Uso de internet, la web y aplicaciones de software para hacer negocios. Dicho de manera más formal, comprende las transacciones comerciales digitales que ocurren entre organizaciones, entre individuos, y entre organizaciones e individuo" (p. 12).

Chaffey (2011, citado en Chaffey \& EllisChadwick, 2014) por su parte indica que: “ $E 1$ comercio electrónico (e-commerce) se refiere a las transacciones financiera $y$ de información realizadas de manera electrónica entre una organización y cualquier tercero con el que tenga tratos" (citado en Chaffey \& Ellis-Chadwick, 2014, p. 20).

También Lu \& Hung (2011) define que: "Conceptualmente, la venta al por menor electrónica puede ser entendida como sinónimo de venta de productos y servicios directamente para el consumidor por medio de un canal electrónico" (citado en Barboza, de Medeiros, de Farias, \& da Costa, 2015, p. 73).

Existen varios tipos de comercio electrónico, presentados en la tabla 1 del cual se ha seleccionado el modelo B2C para la realización de este estudio, el cual tomando como referencia lo que mencionan Laudon and Traver (2014, p. 22) es el tipo más utilizado porque es con el cual se trata de llegar a más consumidores individuales y en este tipo de comercio es donde se encuentra la mayoría de los consumidores, agregando que aunque el modelo $\mathrm{B} 2 \mathrm{C}$ es aun relativamente pequeño, ha crecido exponencialmente desde 1995 y dentro de esta categoría hay muchos tipos de negocio.

En virtud de lo anterior, se toma como referencia a los autores Aziz and Fady (2013, p. 582) quienes presentan el modelo de las etapas del desarrollo del e-Business creado por Earl (2000) ilustrado en la figura 2, quién a su vez indica que la nueva era de negocios coloca al internet como una plataforma para realizar transacciones y una red troncal de negocios, mencionando también que la 
El comercio electrónico como estrategia de negocios para productos de belleza hacia el mercado chileno: Caso de la empresa the well life company

revolución tecnológica de internet afecta a todas las actividades comerciales.

Del análisis anterior, en varias etapas se menciona que para que el comercio electrónico se efectúe, deben de ocurrir compras y ventas, Urueña, Agudo-Peregrina y Hidalgo-Nuchera (2011, citado Zárate, 2015, p. 84) el internet se ha convertido en una fuente de información para las personas que lo utilizan en sus procesos de compras, involucrando así una etapa de concientización y análisis de la información obtenida por los consumidores.

TABLA 1. PRINCIPALES TIPOS DE COMERCIO ELECTRÓNICO

\begin{tabular}{ll}
\hline Tipos de comercio electrónico & Descripción \\
\hline B2C-de negocio a consumidor & Los negocios en línea tratan de llegar a los consumidores individuales. \\
B2B- de negocio a negocio & Los negocios se enfocan en vender a otros negocios, es la mayor forma de comercio electrónico. \\
C2C- de consumidor a & $\begin{array}{l}\text { Ofrece a los consumidores una manera de vender a otros consumidores, con la ayuda de un } \\
\text { generador de mercado en línea como eBay o Craiglist. }\end{array}$ \\
consumidor & Se hace posible mediante redes sociales y relaciones sociales en línea. \\
Comercio electrónico social & Se refiere al uso de dispositivos móviles para realizar transacciones en la web, como teléfonos \\
M-commerce- comercio & inteligentes, computadoras portátiles, netbooks y tabletas. \\
electrónico móvil & Es una forma de comercio electrónico que se enfoca en atraer consumidores con base en la \\
Comercio electrónico local & ubicación geográfica real. \\
\hline
\end{tabular}

Fuente: Elaboración propia con datos de Laudon, Traver, \& Ramos (2014, pp. 21-23)

Chaffey and Ellis-Chadwick (2014) agregan a lo anterior que: "La conveniencia sigue siendo la principal motivación de los compradores en línea, mientras que la preferencia de comprar en las tiendas y no ver la necesidad de comprar en línea son los principales obstáculos para su adopción" (p. 73).

FiguRA 2. MODELO DE LAS ETAPAS DEL DESARROLLO DEL E-BUSINESS DE EARL

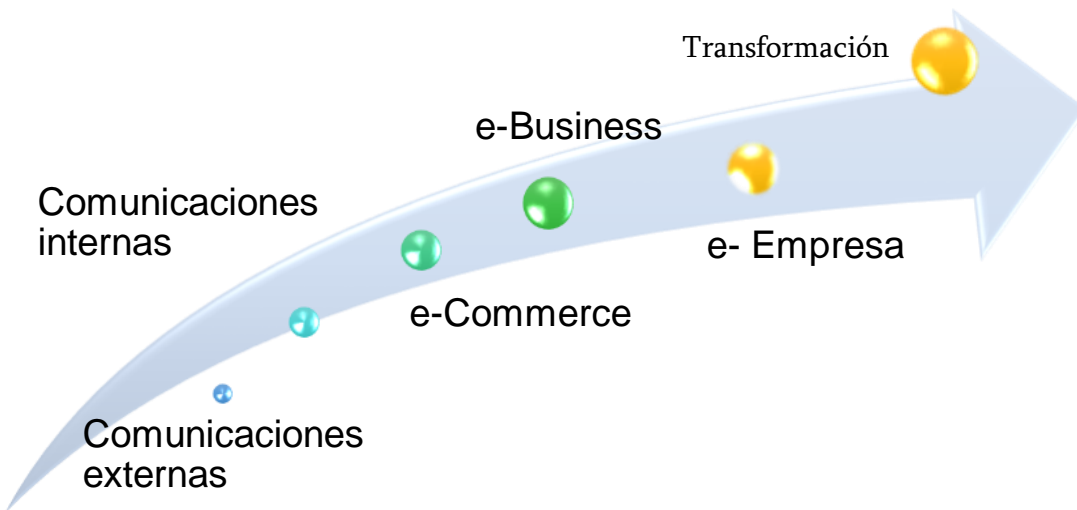

Fuente: Elaboración propia con información de Aziz \& Fady (2013, p. 582)

Bajo esta perspectiva, los usuarios recaudan información usando internet para encontrar lo que le es más conveniente, traduciendo esto a que el cliente busca una propuesta de valor en línea, que de acuerdo al autor mencionado en el párrafo anterior, se entiende como propuesta de valor a la "Declaración de los beneficios en línea que refuerza la propuesta central y distingue la oferta fuera de línea de una organización de la de los competidores" (Chaffey \& EllisChadwick, 2014, p. 14). 
Por su parte Koo, Song, Kim, and Nam (2006) destacan que al utilizar las nuevas tecnologías, la empresa puede conocer las preferencias de los consumidores, lo que facilita la segmentación de mercados, que de igual manera como mencionan Kaplan y Haenlein (2010, citado en Levina \& VilnaiYavetz, 2015) "Entre mayor es la presencia social, mayor es la influencia de la comunicación que las partes tienen en el comportamiento de cada quien" (p. 481).

Lo anterior conlleva a lo mencionado por Chaffey and Ellis-Chadwick (2014, p. 63) quienes indican que el análisis de los clientes puede verse desde dos perspectivas: el análisis de la demanda siendo la primera, que implica entender el contexto en el cual los usuarios se convierten en resultados tácticos y estratégicos y entendiendo el volumen potencial y real que ellos significan; mientras que la segunda perspectiva es la del comportamiento del consumidor digital, en donde se comprenden las características, comportamiento y necesidades de los consumidores, para así desarrollar estrategias y planificación hacia esos segmentos.

Enseguida Palacios et al. (2014) indican que: "La personalización es una estrategia general seguida por las empresas para aumentar la satisfacción del cliente" (p. 228). A su vez Gao et al. (2010) citado en Palacios et al. (2014) describen que: "El perfil del usuario, el modelado de contenidos y filtrado de información como las tres actividades necesarias para una personalización éxito" (p. 228).

Con esa perspectiva do Amaral et al. (2015) denota que la santificación de un producto brinda no lo es todo, sino también los servicios que lo acompañan y cada vez más los clientes quieren hacer valer el dinero usado en sus compras, o sea, están comprando resultados, por tal razón el proceso de calidad de entrega se considera importante, entre otros factores, apuntando que la capacidad de resolver problemas es uno de los más relevantes.

\subsection{Planes de negocios}

Según Stutely (2000, p. 33 y 59) la planificación se entiende como la organización de recursos y acciones que con el fin de lograr determinadas metas habrán de utilizarse y ejecutarse en el futuro, y aunque la planificación sea mínima, aporta seis grandes ventajas básicas, de las cuales se derivan múltiples beneficios. Esas seis ventajas básicas son:

$\Rightarrow$ Suministra una guía de las acciones a seguir.

$\Rightarrow$ Suministra un valioso instrumento de comunicación interna.

$\Rightarrow$ Construye un útil y eficaz método para el autodesarrollo como empresario.

$\Rightarrow$ Constituye un eficaz instrumento de comunicación externa.

$\Rightarrow$ Ayuda a crear una mentalidad interna orientada a la rentabilidad.

$\Rightarrow$ Constituye un eficaz instrumento de control.

Un plan de negocios, desde la perspectiva de Pedraza (2014, p. 5), consta de 10 partes principales principales (Ver figura 3 ):

$\Rightarrow$ Descripción del negocio

$\Rightarrow$ Portafolio de productos y servicios

$\Rightarrow$ Mercado

$\Rightarrow$ Análisis de la competencia

$\Rightarrow$ Procesos y procedimientos de operación

$\Rightarrow$ Organización y el personal estratégico

$\Rightarrow$ Aspectos económicos financieros

$\Rightarrow$ Principales riesgos y estrategias de salida 
El comercio electrónico como estrategia de negocios para productos de belleza hacia el mercado chileno: Caso de la empresa the well life company

$\Rightarrow$ Sistema de seguimiento de la gestión

$\Rightarrow$ Documentos de apoyo y anexos

FIGURA 3. MAPA CONCEPTUAL DE PLAN DE NEGOCIOS

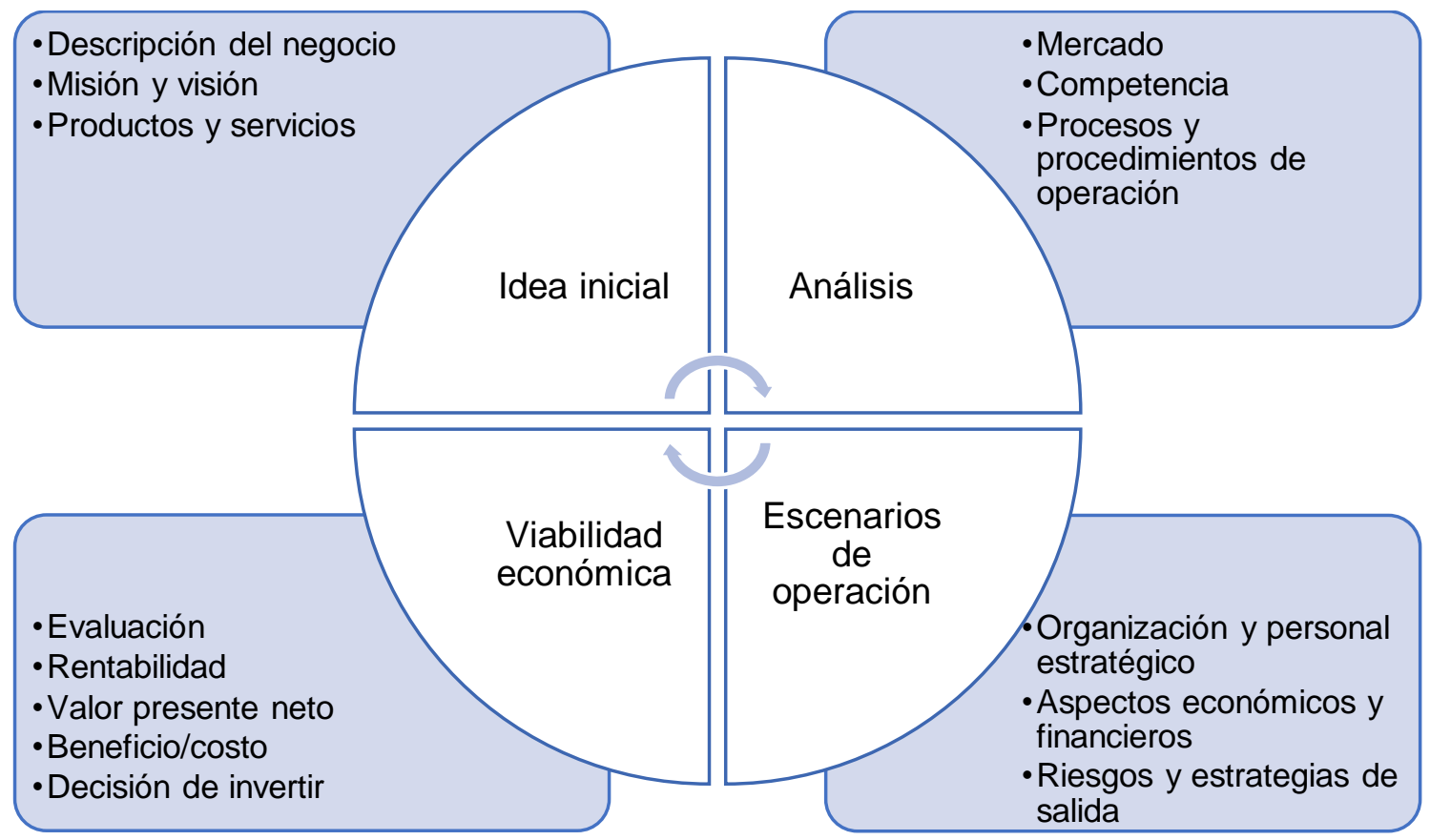

Fuente: Elaboración propia con información de Pedraza (2014, p. 4)

Se contemplaron planes de negocios de diferentes instituciones como base fundamental de este estudio tomando como referencia al de Bancomext porque se consideró el más adecuado al estudio en cuestión, que conlleva los pasos para el desarrollo de la empresa y la exportación de sus productos y que es cimiento para la elaboración del plan de negocios.

\section{METODOLOGÍA}

El paradigma metodológico abordado en esta investigación es el predominantemente cualitativo con datos cuantitativos, porque es más flexible y dio pauta a desarrollar los supuestos planteados, además que por su característica de descriptivo, permitió llevar a cabo una investigación profunda del fenómeno, el cual fue la realización de un plan de negocios para la exportación de productos de belleza al mercado chileno utilizando el comercio electrónico como estrategia, con el cual empresas similares se pueden apoyar en situaciones parecidas.

La destreza metodológica propia del método cualitativo que se utilizó es el estudio de caso único, que como se mencionó anteriormente, no existe información abundante acerca de cómo lograr las exportaciones gracias a la comercialización en línea y el efecto que se tiene en las PYMES. Se determinó el tamaño de muestra por la persona encargada de la empresa, esto debido a que es la persona con conocimiento en el área y experiencia, además de ser el autor intelectual de la idea para la creación del negocio y se apoyó el método seleccionado con una entrevista semiestructurada a realizar a la persona antes indicada de la empresa que facilitó la obtención de 
opiniones, documentos y materiales culturales de la empresa para así realizar un mejor análisis de cómo se encuentra la empresa y la obtención de resultados de la investigación.

Los estudios de casos se pueden realizar y escribir con muchos motivos diferentes que varían desde llegar a generalidades amplias basadas en casos de estudio hasta la presentación de casos individuales (Yin, 2009, p.20).

Ahora bien, de acuerdo con el desarrollo teórico, para los estudios de caso, el desarrollo de la teoría como parte de la fase de diseño es esencial, ya sea que el propósito del estudio de caso resultante sea desarrollar o probar la teoría (Yin, 2009, 35).

The Well Life Company es una empresa catalogada dentro de las microempresas, que tiene como actividad la comercialización de productos de belleza, la cual se constituye como una Sociedad de Responsabilidad Limitada (S. de R. L.) en la ciudad de Culiacán, Sinaloa, cuyo producto principal es el colágeno en crema, que se desea comercializar en México y exportar al mercado chileno. Su fracción arancelaria de acuerdo con el Sistema de Información Arancelaria Vía Internet (SIAVI, 2017) es el 3304.99.99

El objetivo principal de la empresa de este estudio es: "Satisfacer las necesidades de la demanda de productos de belleza de origen natural y optimizar las funciones del sistema biológico generando una mayor calidad de vida", por ende la estrategia principal de entrada es utilizar el modelo de comercio electrónico de Business to Clients (B2C) para la comercialización de los productos de belleza en ambos países directamente a los consumidores, dadas todas las ventajas que éste ofrece, y en segundo lugar también el valor agregado de los productos, siendo éste que gracias al departamento de Investigación y Desarrollo, contiene los mayores índices de colágeno a nivel mundial.

Otra de las estrategias en acción para la exportación de volúmenes altos, es la de enviar los productos por medio de transporte marítimo, debido a que los productos no son perecederos además de que el país chileno también es costero y se considera como una puerta para entrar a países colindantes en Sudamérica. A parte de eso, este estudio demostró que el nicho de mercado al que va dirigido es a mujeres de 30 años a 45 años que habitan en ambos países.

En esta investigación se encontró que las exportaciones de preparados de belleza, de acuerdo con el Banco de México (2016) las exportaciones totales de preparaciones de belleza para piel hacia noviembre de 2015 fueron de 21,486 miles de dólares y en enero de 2016 fueron de 19,014. Sin embargo, se espera que esta tendencia siga en aumento, arrojando que las exportaciones de estos productos hacia Chile sean potenciales además que el consumo de estos en el país meta cada vez van en mayor aumento.

\section{RESULTADOS}

Respuesta a interrogante central: La empresa podrá contar con una oportunidad de mercado, una estructura adecuada en su organización, así como los elementos necesarios para iniciar las exportaciones, dando como resultado un proceso de internacionalización exitoso.

\subsection{Respuesta a interrogante específica 1:}

Se debe tomar en cuenta primeramente la globalización, dado que las empresas que no 
El comercio electrónico como estrategia de negocios para productos de belleza hacia el mercado chileno: Caso de la empresa the well life company

pueden o no quieren participar en ella, están destinadas a fracasar por el hecho de que la competencia sí lo está haciendo aún sin notarlo. En segundo lugar, la selección de un mercado meta correcto, que en este estudio se demuestra que el país Chile es un excelente lugar para incursionar dadas las características y tendencias del consumo de productos de belleza, el poder adquisitivo, así como los altos índices del uso de internet, convirtiéndose en uno de los mercados más atractivos de América Latina, además de los beneficios que podría traer a futuro con la región donde se encuentra. Por otra parte, se toma en cuenta también, la fracción arancelaria, normas de calidad, las barreras arancelarias y no arancelarias necesarias y la documentación solicitada para lograr con éxito las exportaciones.

\subsection{Respuesta a interrogante específica 2:}

El comercio electrónico tiene ventajas enormes, primero porque se evitan los costos fijos de tener un comercio tradicional, la logística por tierra, muchos empleados, la renta de espacios, que se pueden traducir a más publicidad por internet, la cual llega a audiencias increíblemente mayores que de la manera tradicional, asimismo de que no hay barreras geográficas por lo que el negocio puede alcanzar más clientes en diferentes ciudades o países, además de que con las herramientas de internet, la empresa puede trabajar día y noche aunque los empleados no estén laborando en el momento.

\subsection{Respuesta a interrogante específica 3:}

Asegurarse que existen apoyos tanto gubernamentales como privados para impulsar el crecimiento empresas, así como que la empresa esté actualizada en cuanto a las normas necesarias, certificaciones $y$ materia legal, aprovechar las oportunidades y tendencias del mercado y siempre estar preparada para evolucionar y así como siempre estar un paso más adelante que la competencia. Por otro lado, es de suma importancia tener las metas y objetivos bien planteados $y$ tener bien definidas las funciones del equipo de trabajo y que trabajen conjuntamente para lograr un desempeño alto y armonioso.

En el cuerpo de este documento, se demostró el mercado chileno, seleccionado para la exportación de los productos de belleza de origen mexicano, es técnicamente factible, porque se encontró que gracias a su localización geográfica es de fácil acceso, la cultura del uso de los productos que esta empresa desea comercializar es alta, los cuales van dirigidos a un nicho de mercado de mujeres de clase media alta que habitan en ese país y que realizan compras a tiendas tanto nacionales como internacionales a través del internet, ponderando que son aproximadamente $\quad 5,193,562$ mujeres haciéndolo viable.

Tomando en cuenta los planes de la Agenda Conectar 2020 de incrementar el acceso a internet a nivel global, a los crecientes índices de usuarios que utilizan el mismo según el Internet World Stats, el crecimiento del comercio electrónico en México que va a más allá de los 12.2 billones de dólares y al alto índice del desarrollo de las TICs en Chile, se invita tanto al sector gobierno, privado y universidades a incentivar las investigaciones para el desarrollo de las TICs en México, así como el uso de herramientas como el Internet World Stats, el World Trade Map, INEGI, las que ofrece Pro México y entre otras mencionadas en el documento, para que sirva como referencia de los mercados que pueden ser explotados. 
Dentro del apartado financiero del plan de negocios se presentan las ventas correspondientes durante los siguientes 5 años llegando hasta $4,000,000$ pesos promedio de ganancias netas anuales, siendo esto un factor rentable para la empresa.

Retomando la información presentada en el plan, los estados financieros y el estudio de mercado, se puede confirmar que el objetivo principal de éste se ha logrado exitosamente, éstos podrán ser utilizados por la empresa como base fundamental para la operación de esta, tener una visión amplificada del mercado y sus alcances, para de esta manera evitar posibles pérdidas económicas $y$ aprovechar todas las oportunidades posibles.

\section{CONCLUSIONES}

Las personas cada vez invierten más en los cuidados de belleza porque desean sentirse y verse bien, por lo que optan por mejores productos de acuerdo con sus necesidades, es por esa demanda de productos que la empresa en investigación se convierte comercialmente importante. De ahí que se considera lo fundamental que es para cualquier empresa contar con el objetivo de esta investigación, que es un plan de negocios.

Es común que las micro y pequeñas inicien operaciones sin antes haber realizado un estudio de mercado y sin considerar los demás aspectos que estas requieren para que su consolidación sea exitosa, por lo que se considera al plan de negocios un pilar para esencial para que las empresas de todo tipo puedan ser más competitivas tanto a nivel local como global.

Debido a lo anterior, se recomienda a las empresas la elaboración de un plan de negocios para asegurarse que éstas conozcan su viabilidad, por lo que pueden utilizar la presente investigación en las áreas académicas, profesionales y empresariales como ejemplo para la elaboración de este, sobre todo, puede servir como acervo bibliográfico en las relacionadas con carreras de administración, negocios internacionales, comercio electrónico y comercio exterior.

Asimismo, el plan de negocios realizado puede servir como guía para las empresas y emprendedores que deseen incursionar en los mercados internacionales, así como le podría servir a las dependencias gubernamentales del estado de Sinaloa como guía del proceso de exportación.

\section{REFERENCES}

AMIPCI. (2015). Estudio comercio electrónico en México 2015. Asociación mexicana de internet. Recuperado de https://amipci.org.mx/estudios/comercio_electronico/Est udio_de_Comercio_Electronico_AMIPCI_2015_version_ publica.pdf

Aziz, R. A. E., \& Fady, R. (2013). Business improvement using organisational goals, riva technique and e-business development stages: A case study approach. Journal of Enterprise Information Management, 26(5), 577-595. doi:10.1108/JEIM-07-2013-0044

Baier-Fuentes, H., Hormiga, E., Miravitlles, P., \& BlancoMesa, F. (2019). International entrepreneurship: a critical review of the research field. European Journal of International Management, 13(3), 381-412. https://doi.org/10.1504/EJIM.2019.099427

Banco de México. (2016). Exportaciones totales de preparaciones de belleza para piel. Sistema de información económica. Recuperado de http://www.banxico.org.mx/SieInternet/consultarDirecto rioInternetAction.do?accion=consultarCuadro\&idCuadro $=$ CE37\&sector $=1 \&$ locale $=$ es.

Bancomext. Un plan de negocios efectivo, tu llave para exportar como los grandes. Recuperado de https://www.bancomext.com/blogbancomext/pymex/un-plan-de-negocios-efectivo-tullave-para-exportar-como-los-grandes/

Barboza, S. I. S., de Medeiros, F. G., de Farias, H. C. A., \& da Costa, F. J. (2015). Determinantes do comprometimento de consumidores no contexto do varejo virtual. REMark Revista Brasileira de Marketing, 14(1), 72-83. doi: 10.5585/remark.v14i1.2470 


\section{El comercio electrónico como estrategia de negocios para productos de belleza hacia el mercado chileno: Caso de la empresa the well life company}

Barker, M. S, Barker, D. I., Bormann, N., \& Neher, K. (2015). Marketing para medios sociales: Un planteamiento estratégico. México, D.F: Cengage Learning Editores.

Barua, A., Konana, P., Whinston, A., \& Yin, F. (2004). An empirical investigation of net-enabled business value. MIS Quarterly, 28(4), 585-620. doi: 10.2307/25148656

Bergiel, B. J., Bergiel, B. J., \& Bergiel, E. B. (2014). Are you listening to the people that count?. Competition Forum, 12(2), 29

Bernal, E., (2015). Plan de negocios como factor determinante de crecimiento para una comercializadora de productos deportivos, caso de la empresa Feler Euro-Asian. (Tesis de Maestría, Universidad Autónoma de Sinaloa, Culiacán), Sinaloa, México. Recuperado de https://mae.posgrado.fca.uas.edu.mx/images//repositorios/ Libro_Administracin_estratgica.pdf

Cardozo, P. P., Chavarro, A., \& Ramírez, C. A. (2013). La internacionalización de pymes de alta tecnología de Bogotá. Casos de estudio. Panorama, 1(3), 43-64.

Contreras, E. E. M. (2015). La importancia de un plan de negocios de exportación de una empresa de sistemas de riego para el mercado de Costa Rica, Centroamérica. Caso de la empresa Netafim México S.A. de C.V. (Tesis de Maestría, Universidad Autónoma de Sinaloa, Culiacán), Sinaloa, México. Recuperado de https://mae.posgrado.fca.uas.edu.mx/images//repositorios/ Libro_Administracin_estratgica.pdf

Chaffey, D., \& Ellis-Chadwick, F. (2014). Marketing digital estrategia, implementación y práctica. México, D.F.: Pearson educación.

Chiavenato, I. (2014). Introducción a la teoría general de la administración (8a ed.). España: McGraw Hill.

Correa, T., Batista, J., Kassouf, A., \& Dias, T. (2015). Influências do comércio eletrônico B2B no processo de atendimento de pós-vendas no segmento de bens de capital: estudo de caso em uma empresa representante de equipamentos pesados. Revista Brasileira de Marketing, 14(2), 223-236. doi: 10.5585/remark.v14i2.2933

Da Silva, R. O. (2002). Teorías de la administración: Thomson Learning.

David, F. R. (2013). Conceptos de administración estratégica (14 ed.). México D.F.: Pearson Educación.

Dess, G. G., Sánchez, G. A., Lumpkin, T. G., Eisner, A. B., Mejía, R., \& Vargas, Y. M. (2011). Administración estratégica: textos y casos. México: McGraw-Hill.

do Amaral, T. C., de Camargo, J. B. J., Pizzinatto, A. K., \& de Toledo, T. C. D. P. (2015). Influências do Comércio Eletrônico B2B no Processo de Atendimento de PósVendas no Segmento de Bens de Capital: Estudo de Caso em uma Empresa Representante de Equipamentos Pesados. Revista Brasileira de Marketing, 14(2), 223-236
Gonçalves, R., Barroso, J., Varajão, J. E., \& Bulas-Cruz, J. A. (2008). Modelo das iniciativas de comércio eletrónico em organizações portuguesas. Interciencia: Revista de ciencia y tecnología de América, 33(2), 120-128.

González, R. B. (2011). Diferentes teorías del comercio internacional. Información Comercial Española, ICE: Revista de economía, 1(858), 103-118.

Hernández, S. R., Fernández, C. C., \& Baptista, L. P. (2014). Metodología de la investigación. (6a ed.). México D.F.: McGraw-Hill.

Hitt, M. A., Ireland, R. D., \& Hoskisson, R. E. (2015). Administración estratégica: Competitividad y globalización conceptos y casos. México, D.F.: Cengage Learning.

Internet World Stats. (2016). The internet big picture. Recuperado http://www.internetworldstats.com/stats.htm

Koo, C., Song, J., Kim, Y. J., \& Nam, K. (2006). Do e-business strategies matter? The antecedents and relationship with firm performance. Information Systems Frontiers, 9(2-3), 283-296. doi:10.1007/s10796-006-9008-1

Krugman, P. R., Obstfeld, M., \& Melitz, M. J. (2012). Economía Internacional: teoría y política (9 ed.). Madrid, España: Pearson.

Laudon, \& Traver. (2014). E-commerce 2013. Naucalpan, Estado de México. México D. F.: Pearson.

Levina, O., \& Vilnai-Yavetz, I. (2015). E-visibility maturity model: A tool for assessment and comparison of individual firms and sets of firms in e-business. Electronic Commerce Research and Applications, 14(6), 480-498. doi: 10.1016/j.elerap.2015.07.004

Madsen, T. K., \& Servais, P. (1997). The internationalization of born globals: an evolutionary process?. International Business Review, 6(6), 561—583. doi: 10.1016/S09695931(97)00032-2

Nafin. (2008). Clasificación PyMES. Recuperado de http://www.nafin.com/portalnf/content/zproductos-yservicios/programas-empresariales/clasificacionpymes.html

Palacios, L. L., Juste, V. B., Redondo, Y. P., \& Grünhagen, M. (2014). E-business implementation and performance: analysis of mediating factors. Internet Research, 24(2), 223-245. doi:10.1108/IntR-09-2012-0195

Pedraza, Ó. H. R. (2014). Modelo de plan de negocios para la micro y pequeña empresa. (1a Ed.). México: Grupo Editorial Patria.

Porter, M. (2008). Las cinco fuerzas competitivas que le dan forma a la estrategia. Harvard Business Review, 86(1), 58-77.

Romero, B. C. M. (2015). Cambio organizacional para el desarrollo exportador en la mediana empresa hortícola del 


\section{Romero Rubio, Salvador Abraham y Guzmán Lares, Gregorio}

estado de Sinaloa. (Tesis de Doctorado, Universidad de Occidente), Culiacán, Sinaloa, México.

Sanou, B. (2015). Informe sobre Medición de la Sociedad de la Información 2015. Resumen ejecutivo. Recuperado de http://www.itu.int/en/ITUD/Statistics/Documents/public ations/misr2015/MISR2015-ES-S.pdf

Stutely, R. (2000). Plan de negocios: la estrategia inteligente. México D.F.: Pearson Educación.

The Nielsen Company. (2016). A los chilenos les gustan hacer compras online de e-tailers extranjeros. Recuperado de http://www.nielsen.com/cl/es/insights/news/2016/A-loschilenos-les-gustan-hacer-compras-online-de-e-tailersextranjeros.html

Torres, R. G. (2005). Teoría del comercio internacional. México, D.F.: Siglo XXI editores.

Valdez, O. R. S. (2015). Mecardotecnia digital: Estrategia de competitividad en el comercio electrónico de la empresa multinacional. Caso Coppel S.A. de C.V. (Tesis de Maestría, Universidad Autónoma de Sinaloa, Culiacán), Sinaloa, México.

Yin, R. K. (2009). Case study research: design and methods. Applied social research methods. (5a ed.). USA: SAGE Publications.

Zárate, M. L. O. (2015). El uso de Internet en México aplicado a la mercadotecnia en e-Business. (Tesis de Maestría, Instituto Politécnico Nacional, México, D.F.), México D.F., México. 